\title{
Increased expression of miR-148b in ovarian carcinoma and its clinical significance
}

\author{
HUA CHANG $^{1 *}$, XIN ZHOU $^{2 *}$, ZHEN-NING WANG $^{1}$, YONG-XI SONG $^{1}$, \\ FANG ZHAO $^{2}$, PENG GAO $^{1}$, YEUNPO CHIANG ${ }^{1}$ and HUI-MIAN XU ${ }^{1}$ \\ ${ }^{1}$ Department of Surgical Oncology and General Surgery, The First Hospital of China Medical University; \\ ${ }^{2}$ Department of Gynecology and Obstetrics, ShengJing Hospital of China Medical University, Shenyang, P.R. China
}

Received November 10, 2011; Accepted February 10, 2012

DOI: $10.3892 / \mathrm{mmr} .2012 .794$

\begin{abstract}
Ovarian cancer affects females worldwide and is associated with poor patient prognosis. Identification of diagnostic biomarkers and effective therapeutic targets is vital for the diagnosis and treatment of ovarian cancer. Recently, researchers have found that microRNAs play several important roles in carcinogenesis. The purpose of this study was to investigate the relationship between miR-148b expression in human ovarian cancer tissues and clinicopathological features. Seventy-seven ovarian carcinoma tissues and 17 normal ovary tissues were collected from the First Hospital of China Medical University. For quantitative detection of the expression levels of miR-148b, total RNA was extracted and then reverse transcription-polymerase chain reaction was performed. The relationship between miR-148b expression in ovarian cancer and clinicopathological features was analyzed. We found that miR-148b was overexpressed in $92.21 \%(71 / 77)$ of the ovarian cancer samples examined, and overexpression of miR-148b in ovarian cancer tissues was not associated with any of the clinicopathological features of patients with ovarian cancer. Taken together, miR-148b may be involved in the early stage of ovarian carcinogenesis and could be used as an efficient diagnostic biomarker.
\end{abstract}

\section{Introduction}

microRNAs (miRNAs) are endogenous non-coding RNAs, 18-22 nucleotides in length, that modulate gene expression in a post-transcriptional manner (1). Studies have estimated that miRNAs are widely expressed in various species and

Correspondence to: Dr Zhen-Ning Wang, Department of Surgical Oncology and General Surgery, The First Hospital of China Medical University, Shenyang 110001, P.R. China

E-mail: josieon826@yahoo.com.cn

\section{*Contributed equally}

Key words: microRNA, ovarian cancer, clinicopathological features, miR-148b tissues, which indicate that miRNAs play an important role in different biological processes, such as cell proliferation during development (2), cell growth (3), invasion (4) and apoptosis, as well as carcinogenesis, differentiation (5-7), biogenesis, transcription, signal transduction, cell cycle, neurogenesis and fat metabolism.

Ovarian cancer is one of the most common gynecological malignancies and the leading cause of death among females (8). Epithelial ovarian cancer, which accounts for $90 \%$ of ovarian cancers, is divided into several histological subgroups, such as serous, mucinous, endometrioid, clear cell, Brenner and undifferentiated carcinomas $(9,10)$. Ovarian cancer manifests few symptoms in its early stage, for which trans-vaginal ultrasound and serum CA-125 remain poor tests for diagnosing the disease (11). Therefore, most patients are diagnosed at an advanced stage and have a 5-year survival rate of only 20-25\% $(12,13)$. Sensitive and specific biomarkers for the early detection of ovarian cancer are urgently required to reduce the high mortality of the disease. The overall 5-year survival rate can be improved to greater than $90 \%$ if ovarian cancer is confined to the ovary at the time of diagnosis.

Many miRNAs are implicated in ovarian cancers, but studies involving a large number of cases concerning the role of miR-148b in such cancers are lacking. Our previous study showed that miR-148b was down-regulated in gastrointestinal cancer (14).

Here, for the first time, we examined the expression of miR-148b in a large number of ovarian cancers. We found that miR-148b was up-regulated in cancer tissues compared to normal ovarian tissues. We also discussed the associations between increased expression of miR-148b and clinicopathological characteristics of epithelial ovarian cancer.

\section{Patients and methods}

Ethical approval of the study protocol. The present study was approved by the Research Ethics Committee of China Medical University (Shenyang, China). Written informed consent was obtained from all patients.

Patients. Seventy-seven ovarian cancer tissues from patients were collected at The First Affiliated Hospital of China Medical University. The median patient age was 54 years 
(range 31-80). Tumor and normal tissue samples were freshly obtained from resected specimens, and included 17 samples of normal ovarian tissue and 77 epithelial ovarian tumors. One section of each specimen was stained with H\&E for histopathological evaluation. All tumors were staged according to the International Federation of Gynecology and Obstetrics (FIGO) standards.

Total RNA extraction. Total RNA and enrichment of small RNA from fresh samples was isolated using the mirVana miRNA Isolation kit (Ambion, Austin, TX, USA) according to the manufacturer's instructions, and then stored at $-70^{\circ} \mathrm{C}$ until use. Total RNA from fresh cultured cells was carried out with TRIzol reagent (Invitrogen, Karlsruhe, Germany) following the manufacturer's protocol. The concentration and purity of RNA were controlled by UV spectrophotometry (A260/A280 $>1.9$ ) using a Nano-Photometer UV/Vis spectrophotometer (Implen, Schatzbogen, Munich, Germany).

Polyadenylation and reverse transcription-polymerase chain reaction (RT-PCR). Total RNA was polyadenylated with ATP by Escherichia coli poly(A) polymerase (E-PAP) at $37^{\circ} \mathrm{C}$ for 30 min following the manufacturer's instructions for a Poly(A) Tailing kit (Ambion) (15). cDNA was generated using the PrimeScript ${ }^{\mathrm{TM}}$ RT reagent kit (Perfect Real-Time; Takara Bio, Kyoto, Japan). After extraction with phenol-chloroform and precipitation with ethanol, RNA was dissolved in diethyl pyrocarbonate-treated water and reverse transcribed with the PrimeScript RT reagent kit (Perfect Real-Time).

Real-time PCR. Real-time RT-PCR method was used to assess the expression levels of miR-148b with Express $S Y B R^{\circledR}$ GreenER qPCRs supermix Universal kit (Invitrogen) on a Rotor-gene 6000 system (Qiagen, Valencia, CA, USA). U6 RNA was used as an endogenous reference for normalizing the expression levels of miR-148b. Initially, we calculated a ${ }^{\Delta} \mathrm{Ct}$ (target-reference), which is equal to the difference between threshold cycles for miR-148b (target) and those for U6 RNA (reference). The fold-change between cancer tissues and normal ovary tissue control for miR-148b was calculated with the $2^{\Delta \Delta} \mathrm{Ct}$ method, in which ${ }^{\Delta \Delta} \mathrm{Ct}={ }^{\Delta} \mathrm{Ct}$ (target-reference in tumor samples) - ${ }^{\Delta} \mathrm{Ct}$ (target-reference in normal samples). The relative expression levels of miRNAs in cancer compared to their non-tumorous controls were calculated using the method of $2^{-\Delta \Delta} \mathrm{Ct}$ (16). The value of the relative expression ratio $>1.0$ was considered as high expression in cancer relative to the normal sample (17). Each sample was measured in triplicate and repeated three times. The investigators were blinded to the results of the clinical and pathological diagnoses.

Statistical analysis. Statistical analysis was performed using the Statistical Program for Social Sciences (SPSS) software 16.0 (SPSS Inc., Chicago, IL, USA). The Student's t-test was used to analyze the differences in miR-148b expression between the tumor and normal tissues. Correlations between miRNA expression and clinicopathological features were analyzed by a non-parametric test: Mann-Whitney U test between two groups and Kruskal-Wallis test for three or more groups. $\mathrm{P}<0.05$ was considered to indicate statistical significance.

\section{Results}

miR-148b is overexpressed in ovarian cancer tissues compared to normal ovary tissues. From the results of real-time quantitative RT-PCR, we found that miR-148b was overexpressed in $92.21 \%$ (71/77) of the patients with ovarian cancer compared to normal tissues (Fig. 1). The ${ }^{\mathrm{C}} \mathrm{Ct}$ value of miR-148b in the cancer tissues ranged from 7.68 to 11.14 , and that in normal tissues from 11.13 to 12.59 (Fig. 2). The average ${ }^{\Delta} \mathrm{Ct}$ value of miR-148b in the cancer tissues was 9.41, while that in the normal tissues was 11.86 ( $\mathrm{P}<0.001$; Fig. 2).

Expression levels of miR-148b are not associated with clinicopathological features of patients with ovarian cancer. We next performed a preliminary analysis to identify whether miR-148b expression is associated with clinicopathological features using a non-parametric test. No association was found between miR-148b expression levels and clinicopathological features, such as age, FIGO stage, histological subtypes and degree of differentiation (Table I).

We subsequently evaluated the relationship between miR-148b expression and the response to chemotherapy according to RECIST criteria. Among all 77 patients, 49 receiving chemotherapy were followed up. Thirty-one patients showed complete response (CR) to chemotherapy, and 18 progressive disease (PD); no significant difference was noted (Table I). We used the Kaplan-Meier estimator to evaluate the significance of miR-148b and short-term survival time; however, log-rank P-value was 0.98.

\section{Discussion}

The discovery that miRNAs are regulated at different levels in normal vs. malignant tissues offers the possibility of identifying new methods for diagnosing ovarian cancer at early stages. Iorio et al (18) analyzed 69 ovarian epithelial carcinomas and 15 normal ovarian tissue sections; they identified 4 up-modulated and 25 down-modulated miRNAs. Moreover, this study also identified miRNAs which were correlated with specific ovarian cancer biopathological features, such as histotype, lymphovascular and organ invasion. Resnick et al (19) reported that miRNA-21, -29a, -92, -93 and -126 were overexpressed in the serum of ovarian cancer patients compared to controls. This indicates that the serum miRNA levels of the patients may be used to potentially diagnose ovarian cancer. Additionally, miRNA-21, -92 and -93 were also overexpressed in patients with normal CA125 levels. This suggests that miRNA-21, -92 and -93 are known oncogenes with therapeutic and biomarker potential. Yang et al (20) studied 36 miRs in normal ovarian cells and epithelial ovarian tumors; miR-199a*, miR-214, miR-200a and miR-100 were the most highly differentially expressed candidates and their expression was associated with high-grade and late-stage tumors. Our previous study also found that the expression of miR-148a and miR-152 was altered in ovarian cancer and was related to cell proliferation (21).

The miR-148b gene is located at $12 q 13.13$. Its mature sequence, which has been confirmed by experimental and microarray methods, is: UCAGUGCAUCACAGAACUUUGU. To date, little is known about its function. Zhao et al (22) found 


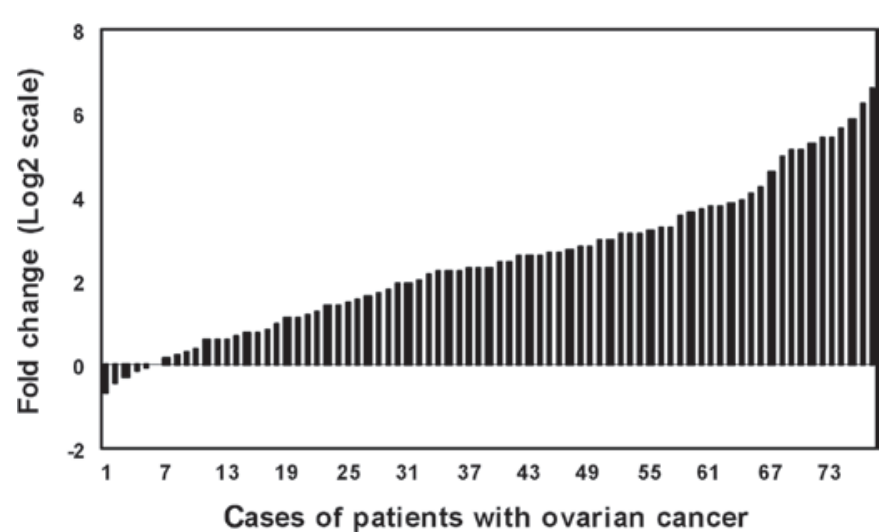

Figure 1. Expression of miR-148b in ovarian cancer patients. miR-148b expression levels were quantified using SYBR Green real-time PCR. Data are presented as the $\log 2$ fold change of ovarian cancer relative to normal tissues. Each sample was analyzed in triplicate and experiments were repeated three times.

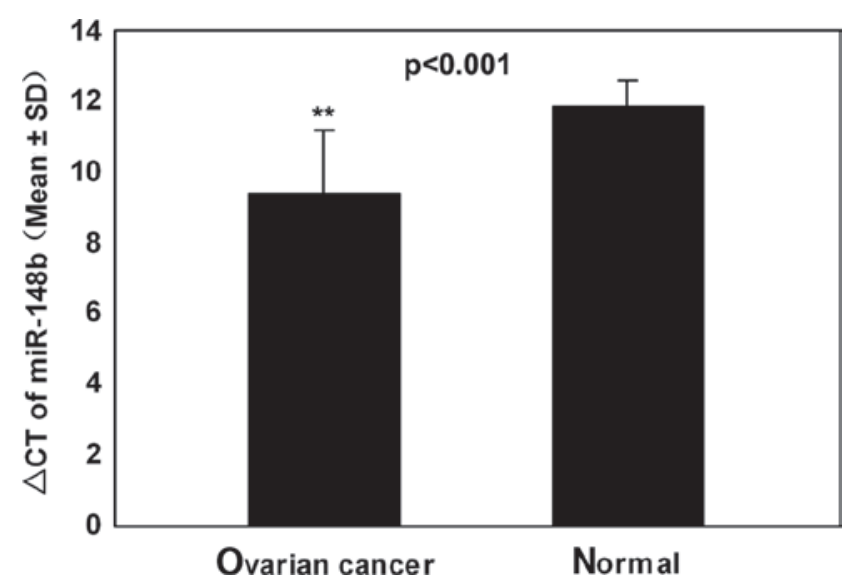

Figure 2. Expression of miR-148b in ovarian cancer tissues compared to normal ovarian tissues. miR-148b expression levels were normalized by U6 RNA. $\Delta$ CT $=$ CT miR-148b - CT U6 RNA. The $\Delta$ CT of miR-148b was significantly lower in ovarian cancer tissues than normal tissues $(\mathrm{P}<0.001$, independent-sample t-test).

that miR-148b was down-regulated in a hepatic cancer cell line compared to a hepatic cell line. Zhang et al (23) reported marked up-regulation of 25 serum miRNAs in esophageal squamous cell carcinoma (ESCC) patients compared to healthy individuals. RT-qPCR analysis identified a profile of 7 serum miRNAs, including miR-148b as ESCC biomarkers.

Liu et al (24) reported that three members of the miRNA-148 family, miR-148a, miR-148b and miR-152, are negative regulators of the innate response and $\mathrm{Ag}$-presenting capacity of dendritic cells (DCs). miRNA-148/152 acts as a fine-tuner in regulating the innate response and Ag-presenting capacity of DCs, which may contribute to immune homeostasis and immune regulation.

Yet, the detailed expression pattern and potential role of miR-148b in ovarian cancer has never been determined. In the present study, we compared miR-148b expression levels in ovarian cancer and normal tissues. By real-time RT-PCR, we found that miR-148b was significantly overexpressed in ovarian cancer compared to normal tissues. Among the 77 samples assessed, miR-148b was upregulated in 71 samples and downregulated in 6 cases. The result suggests
Table I. Correlation between the expression of miR-148b and clinicopathological features in patients with ovarian cancer.

\begin{tabular}{lcc}
\hline & No. & miR-148b \\
\hline Age (years) & & \\
$\leq 54$ & 17 & $5.93(2.51-11.57)$ \\
$>54$ & 60 & $5.00(1.82-12.31)$ \\
P-value & & 0.713 \\
FIGO stage & & \\
I & 16 & $8.22(2.34-14.2)$ \\
II & 5 & $5.40(3.32-24.75)$ \\
III & 48 & $4.76(1.70-9.22)$ \\
IV & 8 & $5.39(3.21-11.68)$ \\
P-value & & 0.609 \\
Histological subtypes & & \\
Serous & 57 & $4.90(11.99-13.21)$ \\
Mucinous & 2 & $1.44(0.94-1.94)$ \\
Endometrioid & 3 & $4.96(1.54-7.51)$ \\
Clear cell & 7 & $9.40(12.98-33.96)$ \\
Other & 8 & $0.19(0.13-0.65)$ \\
P-value & & 0.585 \\
Grade & & \\
Grade 1 & 44 & $4.93(2.20-9.28)$ \\
Grade 2 & & $5.21(11.99-12.71)$ \\
Grade 3 & 4 & $18.38(1.40-46.39)$ \\
Grade 4 & 1 & 11.46 \\
P-value & & 0.818 \\
Response & & \\
CR & & $5.89(2.21-13.66)$ \\
PD & & $5.77(2.07-13.90)$ \\
P-value & & 0.803 \\
\hline
\end{tabular}

${ }^{a}$ Median value of relative expression, with the $25-75$ th percentile in parenthesis. ${ }^{b}$ Forty-nine patients were followed up. CR, complete response; PD, progressive disease.

that elevated miR-148b is a frequent event in human ovarian cancer tissues. While miR-148b increased in ovarian cancer and decreased in hepatic cancer, these differences observed with the same miRNA in different tissues suggest that a single miRNA may have multiple targets; the targets of the miRNA having different functions. For example, the miR-17 cluster was amplified in human non-small cell lung cancer (25), whereas the cluster was frequently deleted in hepatocellular carcinoma (26).

Microarray analysis recently confirmed that ovarian carcinoma of different histotypes (serous, mucinous, endometrioid and mixed) exhibits alteration of different pathways, probably reflecting the gene expression pattern of the organ of origin. Considering that ovarian epithelial carcinomas occur as different histological subtypes characterized by distinct morphologic and molecular genetic alterations, we aimed to compare the miR-148b expression of each of them with normal ovary tissue to evaluate whether miRNA expression is different in distinct histotypes of ovarian carcinomas. However, no statistically significant associations were observed. 
We next performed analysis to identify whether miR-148b expression is associated with other clinicopathological features. We found that overexpression of miR-148b was not associated with poor prognosis of patients with ovarian cancer (Table I). Based on our observations of miR-148b expression levels and clinical analysis, we conclude that miR-148b may play a key role during the early-stage of ovarian carcinogenesis, but alone is not enough to affect or contribute to tumor progression. We found that miR-148b is potentially an efficient biomarker for the diagnosis of ovarian cancer.

Studies demonstrate that many factors regulate the expression of miRNAs, including inactivation by deletion or mutation and altered DNA methylation. In colorectal carcinoma, Han et al found that the expression of approximately $10 \%$ of miRNAs was regulated by DNA methylation, and partial methylation reduction was not sufficient for miRNA re-expression. Iorio et al (18) found that the levels of miR-21, miR-203 and miR-205 were up-modulated in ovarian carcinomas compared to normal tissues, and they were significantly increased in OVCAR3 cells after 5-aza-2'-deoxycytidine demethylating treatment. After the treatment of demethylating agent 5-aza-2'-deoxycytidine, re-expression of miR-148 and miR-152 was observed in various breast cancer cell lines. Therefore, we speculated that demethylation may be one of the mechanisms of up-regulation of miR-148b in ovarian cancer. However, this requires further investigation.

In conclusion, the finding that miR-148b was overexpressed in ovarian cancer suggests that miR-148b may be used as a potential diagnostic biomarker for ovarian cancer.

\section{Acknowledgements}

This study was supported by the National Science Foundation of China (nos. 81172370, 81101990 and 30972879).

\section{References}

1. Bartel DP: MicroRNAs: genomics, biogenesis, mechanism, and function. Cell 116: 281-297, 2004.

2. Chiang Y, Song Y, Wang Z, et al: Aberrant expression of miR-203 and its clinical significance in gastric and colorectal cancers. J Gastrointest Surg 15: 63-70, 2011.

3. Brennecke J, Hipfner DR, Stark A, Russell RB and Cohen SM: Bantam encodes a developmentally regulated microRNA that controls cell proliferation and regulates the proapoptotic gene hid in Drosophila. Cell 113: 25-36, 2003.

4. Mori Y, Ishiguro H, Kuwabara Y, et al: MicroRNA-21 induces cell proliferation and invasion in esophageal squamous cell carcinoma. Mol Med Report 2: 235-239, 2009.

5. Tran N, McLean T, Zhang X, et al: MicroRNA expression profiles in head and neck cancer cell lines. Biochem Biophys Res Commun 358: 12-17, 2007.

6. Calin GA and Croce CM: MicroRNA-cancer connection: the beginning of a new tale. Cancer Res 66: 7390-7394, 2006.
7. Chen CZ, Li L, Lodish HF and Bartel DP: MicroRNAs modulate hematopoietic lineage differentiation. Science 303: 83-86, 2004.

8. Zhang L, Huang J, Yang N, et al: microR NAs exhibit high frequency genomic alterations in human cancer. Proc Natl Acad Sci USA 103: 9136-9141, 2006.

9. Seidman JD and Kurman RJ: Pathology of ovarian carcinoma. Hematol Oncol Clin North Am 17: 909-925, vii, 2003.

10. Shih Ie M and Kurman RJ: Ovarian tumorigenesis: a proposed model based on morphological and molecular genetic analysis. Am J Pathol 164: 1511-1518, 2004.

11. Olivier RI,Lubsen-Brandsma MA, Verhoef S and van Beurden M: CA125 and transvaginal ultrasound monitoring in high-risk women cannot prevent the diagnosis of advanced ovarian cancer. Gynecol Oncol 100: 20-26, 2006.

12. du Bois A, Quinn M, Thigpen T, et al: 2004 consensus statements on the management of ovarian cancer: final document of the 3rd International Gynecologic Cancer Intergroup Ovarian Cancer Consensus Conference (GCIG OCCC 2004). Ann Oncol 16 (Suppl 8): viii7-viii12, 2005.

13. Edwards BK, Brown ML, Wingo PA, et al: Annual report to the nation on the status of cancer, 1975-2002, featuring population-based trends in cancer treatment. J Natl Cancer Inst 97: 1407-1427, 2005

14. Song YX, Yue ZY, Wang ZN, et al: MicroRNA-148b is frequently down-regulated in gastric cancer and acts as a tumor suppressor by inhibiting cell proliferation. Mol Cancer 10: 1-13, 2011.

15. Shi R and Chiang VL: Facile means for quantifying microRNA expression by real-time PCR. Biotechniques 39: 519-525, 2005.

16. Livak KJ and Schmittgen TD: Analysis of relative gene expression data using real-time quantitative PCR and the 2(-Delta Delta C(T)) Method. Methods 25: 402-408, 2001.

17. Wang CJ, Zhou ZG, Wang L, et al: Clinicopathological significance of microRNA-31, -143 and -145 expression in colorectal cancer. Dis Markers 26: 27-34, 2009.

18. Iorio MV, Visone R, Di Leva G, et al: MicroRNA signatures in human ovarian cancer. Cancer Res 67: 8699-8707, 2007.

19. Resnick K, Alder H, Hagan J, Richardson D, Croce C and Cohn D: The detection of differentially expressed microRNAs from the serum of ovarian cancer patients using a novel real-time PCR platform. Gynecol Oncol 112: 55-59, 2009.

20. Yang H, Kong W, He L, et al: MicroRNA expression profiling in human ovarian cancer: miR-214 induces cell survival and cisplatin resistance by targeting PTEN. Cancer Res 68: 425-433, 2008.

21. Zhou X, Zhao F, Wang ZN, et al: Altered expression of miR-152 and miR-148a in ovarian cancer is related to cell proliferation. Oncol Rep 27: 447-454, 2012.

22. Zhao Y, Jia HL, Zhou HJ, et al: Identification of metastasisrelated microRNAs of hepatocellular carcinoma in hepatocellular carcinoma cell lines by quantitative real time PCR. Zhonghua Gan Zang Bing Za Zhi 17: 526-530, 2009 (In Japanese).

23. Zhang C, Wang C, Chen X, et al: Expression profile of microRNAs in serum: a fingerprint for esophageal squamous cell carcinoma. Clin Chem 56: 1871-1879, 2010.

24. Liu X, Zhan Z, Xu L, et al: MicroRNA-148/152 impair innate response and antigen presentation of TLR-triggered dendritic cells by targeting CaMKIIalpha. J Immunol 185: 7244-7251, 2010.

25. Hayashita Y, Osada H, Tatematsu Y, et al: A polycistronic microRNA cluster, miR-17-92, is overexpressed in human lung cancers and enhances cell proliferation. Cancer Res 65: 9628-9632, 2005.

26. Lin YW, Sheu JC, Liu LY, et al: Loss of heterozygosity at chromosome 13q in hepatocellular carcinoma: identification of three independent regions. Eur J Cancer 35: 1730-1734, 1999. 